\title{
Inhibition of Carious Lesions in vitro around Gallium Alloy Restorations by Fluoride Releasing Resin-ionomer Cement
}

\author{
Michihiro NAGAMINE, Nasman NUR ALIM, Toshiyuki ITOTA, \\ Yasuhiro TORII, Michal STANINEC ${ }^{1}$ and Kiyoshi INOUE \\ Department of Operative Dentistry, Okayama University Dental School, \\ 2-5-1 Shikata-cho, Okayama 700-8525, Japan \\ ${ }^{1}$ Department of Restorative Dentistry, \\ University of California San Francisco, School of Dentistry, \\ 707 Parnassus Ave., San Francisco, California 94143-0758
}

Received October 15, 1998/Accepted December 10, 1998

\begin{abstract}
A new fluoride releasing resin-ionomer cement was used for bonding of gallium alloy restorations in vitro. Etching, priming, and fluoride releasing resin-ionomer cement were used in the experimental group (ARG), prior to placement of the gallium alloy restorations. Three different controls were used: gallium alloy only (G), no etching, fluoride releasing resin-ionomer cement, gallium alloy ( $R G$ ), etching, priming, non-fluoride cement and gallium alloy (ACG). The mean shear bond strengths of ARG group to enamel and dentin were higher than those of the three control groups. Artificial secondary caries lesions around the restorations in the experimental group and the control groups were produced, using a strep. mutans culture. The microradiographs were examined for presence of a caries inhibition zone near the restoration. Caries inhibition zones were clearly detected around RG and ARG, but not around G and ACG. The results indicate that the fluoride releasing resin-ionomer cement provided good adhesion and caries inhibition in enamel and dentin.
\end{abstract}

Key words: Secondary caries in vitro, Gallium alloy, Fluoride releasing resin-ionomer cement

\section{INTRODUCTION}

Secondary caries is one of the main reasons for replacement of amalgam restorations. Amalgam restorations were previously dependent upon mechanical retention, but can now be performed adhesively. However, the margins of amalgam fillings frequently partially fracture, and the width of the gap between amalgam and tooth gradually increases with time. Many investigators have reported that oral microorganisms penetrate into the ditched margins and the gap, resulting in secondary caries with outer lesions and wall lesions ${ }^{1-3)}$.

Recently, improvements in bonding systems have resulted in increased bond strength between amalgam and tooth $^{4)}$, and improved resistance to microleakage $\mathrm{e}^{5)}$. A previous study reported that the adhesive amalgam technique used with a resin cement inhibited penetration of wall lesions ${ }^{6)}$. The advantages of bonding systems include conservation of tooth structure, and increased retention of amalgam restorations and possibly prevention of marginal fracture.

Fluoride releasing materials are known to inhibit secondary caries; conventional 
and resin-modified glass ionomer restorative materials protect the marginal tooth structure from acid attack ${ }^{7)}$. Glass ionomer lining materials have been used under amalgam restorations because they release fluoride and have both the ability to bond to tooth structure and a low coefficient of thermal diffusion. Recently, resinmodified glass ionomer cements and Bis-GMA based filled resin cements containing almino fluoro-silicate glass, called "fluoride releasing resin-ionomer cements" in this study, have been developed for use as a cavity liner and a luting cement. The use of resin-modified glass ionomers and fluoride releasing resin-ionomer cements as a liner remarkably enhanced the retention of amalgam restorations compared with unlined restorations ${ }^{8,9}$. However, it is not yet clear whether using such a fluoride releasing resin-ionomer cement as a liner provides protection against secondary caries.

Dental amalgam contains mercury, which has raised concerns about possible ill effects on health ${ }^{10)}$. Gallium alloy has been developed to replace mercury-based amalgam, and possesses satisfactory physical properties compared with amalgam for clinical use ${ }^{11)}$. We therefore used gallium alloy as the restorative material in this study.

The purpose of this in vitro study was to test a new fluoride releasing resinionomer cement for inhibition of secondary caries around gallium alloy restorations. We evaluated the shear bond strengths to tooth substrates and observed the presence of a caries inhibition zone adjacent to the restorative materials by microradiography. Moreover, the distributions of calcium and fluoride at the demineralized areas in the caries inhibition zones were compared with those of the intact areas of tooth substrates around restorative materials by electron probe microanalysis.

\section{MATERIALS AND METHODS}

Materials used in this study

Table 1 summarizes the materials used in this study. After the tooth surfaces were treated by the following procedures, gallium alloy (GA) was placed on each specimen. 1. ARG: The tooth surfaces were etched with $10 \%$ phosphoric acid ( $\mathrm{AE}$ ) for 10 sec., rinsed for $10 \mathrm{sec}$., then excess water was gently removed using an air spray, leaving a moist surface. Next, five coats of primer (ABP) were applied and dried, followed by a thin layer of freshly mixed fluoride releasing resin-ionomer cement (RES).

2. G: The tooth surfaces were rinsed with water and dried only, but they were not treated with any agent.

3. RG: The tooth surfaces were merely lined with RES without etching and priming.

4. ACG: The tooth surfaces were etched with $A E$, coated with $A B P$, and then lined with a mixed non-fluoride releasing resin cement $(A B C)$.

\section{Shear bond strength measurement}

Bovine extracted lower incisors were cut with a diamond saw. After embedding the teeth in an epoxy resin, the labial flat enamel and dentin surfaces were ground with \#600-grit silicon carbide paper under running water. The surfaces were treated using 
Table 1 Materials used in this study

\begin{tabular}{|c|c|c|c|}
\hline Material & Code & Batch No. & Ingredient \\
\hline \multicolumn{4}{|l|}{ Adhesive system } \\
\hline ALL-BOND $2^{1}$ & $\mathrm{AB} 2$ & & \\
\hline All-Etch & $\mathrm{AE}$ & 059024 & $10 \%$ phosphoric acid \\
\hline PRIMER A & $\mathrm{ABP}$ & 059094 & $2 \%$ NTG-GMA ${ }^{3}$ \\
\hline PRIMER B & & 059104 & $16 \% \mathrm{BPDM}^{4}$ \\
\hline \multicolumn{4}{|l|}{ Resin cement } \\
\hline ALL-BOND C \& B & $\mathrm{ABC}$ & & \\
\hline \multicolumn{4}{|l|}{ LUTING CEMENT $^{1}$} \\
\hline BASE & & 089244 & Bis-GMA based filled resin \\
\hline CATALYST & & 089234 & \\
\hline \multicolumn{4}{|l|}{ Resin-ionomer cement } \\
\hline RESINOMER $^{1}$ & RES & & Bis-GMA based filled resin \\
\hline BASE & & 019165 & containing sodium-calcium- \\
\hline CATALYST & & 109314 & almino-fluorosilicate glass \\
\hline \multicolumn{4}{|l|}{ Restorative material } \\
\hline $\begin{array}{l}\text { Gallium alloy } \\
\text { GF } \mathbb{I}^{2}\end{array}$ & GA & 9630011107 & Gallium alloy containing $2 \% \mathrm{Pd}$ \\
\hline $\begin{array}{l}\text { 1: Bisco, Inc., Itasca, II } \\
\text { 2: Tokuriki Honten, Tol } \\
\text { 3: N-tolyglycine glycidy } \\
\text { 4: Biphenyl dimethacryl }\end{array}$ & $\begin{array}{l}\mathrm{L}, \mathrm{US} \\
\text { kyo, J } \\
l \text { metl } \\
\text { late }\end{array}$ & hapan & \\
\hline
\end{tabular}

the above-mentioned four procedures. The areas for bonding were demarcated with adhesive tape leaving a hole $3.8 \mathrm{~mm}$ in diameter. Polyethylene tubes with an inner diameter of $3.8 \mathrm{~mm}$ and a height of $2.0 \mathrm{~mm}$ were set on the specimens, and freshly mixed GA was condensed into each tube. After storing the specimens in distilled water at $37^{\circ} \mathrm{C}$ for 24 hours, the shear bond strength was measured using a universal testing machine (AGS-10kND, Shimadzu, Kyoto, Japan) at a cross-head speed of 0.5 $\mathrm{mm} / \mathrm{min}$. The number of specimens was six for each group.

\section{Sample preparation for caries inhibition test}

Twenty four freshly extracted human first upper permanent premolars, free of caries and other defects, were selected for in vitro secondary caries procedures. The sample teeth were randomly divided into three groups of eight and carefully cleaned. The roots were cut off and the pulp tissue was removed. Two class 5 cavities were prepared at the cementoenamel junction on the facial and lingual surfaces of each tooth using a high-speed diamond point with water coolant. The depth of each cavity was standardized at $1.5-2.0 \mathrm{~mm}$ and the width occlusogingivally at $2 \mathrm{~mm}$. The specimen groups of this study are shown in Fig. 1. The experimental cavities (ARG) were filled using AB2 and RES, followed by condensation of GA. Three different controls were devised, using the following procedures: 1.(G); GA only, 2.(RG); no etching and priming, RES and GA, 3.(ACG); AB2, ABC and GA.

The restorations were polished with a white carborundum point under water spray after storage at $37^{\circ} \mathrm{C}, 100 \%$ humidity for 24 hours. The teeth were 

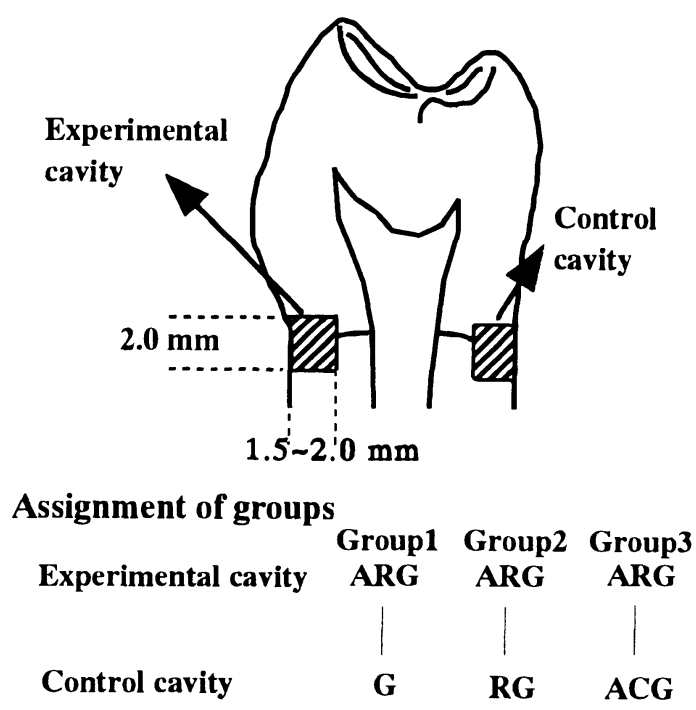

Fig. 1 Diagram of cavity treatments of the three groups.

ARG: Lining with AB 2 \& RES

G: No lining

RG: Lining with RES

ACG: Lining with AB2 \& ABC

thermocycled 500 times between $4^{\circ} \mathrm{C}$ and $60^{\circ} \mathrm{C}$ and were stored at $37^{\circ} \mathrm{C}, 100 \%$ humidity for 28 days.

\section{In vitro secondary caries procedures}

The tooth surfaces were coated with a nail varnish except for a peripheral area of about $0.5 \mathrm{~mm}$ around the margins of the restorations. The specimens were mounted on plastic tubes and sterilized with an ethylene-oxide gas. They were inserted into an artificial caries chamber ${ }^{6,7)}$ and suspended in sterilized brainheart infusion broth (Difco Laboratories, Detroit, MI, USA) containing $0.5 \%$ yeast extract and $1 \%$ sucrose. Hanks' BSS (Nacalai tesque, Kyoto, Japan) was poured into each tube. The broth was inoculated with Streptococcus mutans IFO 13955 and incubated at $37^{\circ} \mathrm{C}$. The medium in the chamber was replaced every 3-4 days and Hanks' BSS in the tube was changed every 7 days. After incubation for 21 days, the specimens were removed and embedded in an epoxy resin.

\section{Examination of caries inhibition zones by microradiography}

The teeth were cut longitudinally through both restorations, and ground sections about $60-80 \mu \mathrm{m}$ in thickness were prepared from each specimen. A contact microradiograph was taken with soft X-ray (Softex CMR-2, Softex Co., Tokyo, Japan) at $16 \mathrm{kVp}, 3 \mathrm{~mA}$ and was observed with a light microscope at $100 \mathrm{x}$. The microradiographs were 

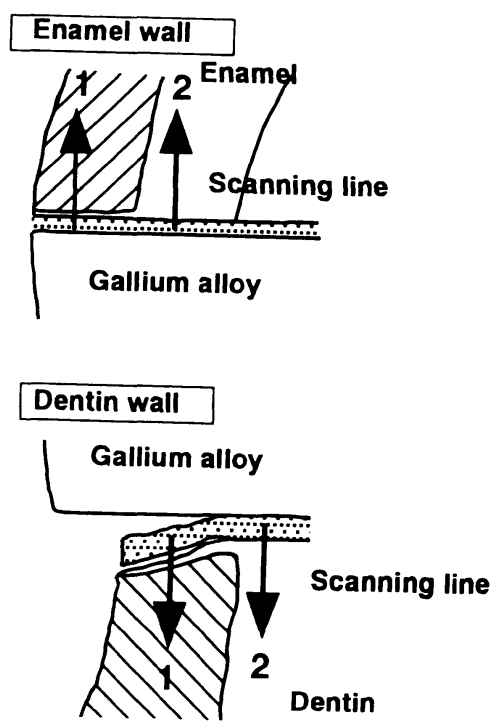

Fig. 2 Sites of scanning lines by electron probe microanalysis.

Arrow 1 shows the scanning line at the demineralized area in the lesion. Arrow 2 shows the scanning line at the intact area underneath the lesion.

examined for presence of radiopacity zones adjacent to the restorations. The zones exhibited small signs of caries inhibition, even in the $G$ control cavity group. We classified the zones at the enamel and dentin lesions adjacent to restorations as follows: zones which were clearly radiopaque and were observed up to the outer surfaces as strongly inhibited; zones which were slightly radiopaque as moderately inhibited; and zones which could not be discerned as not inhibited. The classified data in each group was analyzed with a simple Chi-square analysis at the $\mathrm{P}=0.05$ level.

\section{Microanalysis for calcium and fluoride distribution in tooth substrates}

One section from each of the experimental and control groups was polished, covered by a thin layer of carbon and examined by an electron probe apparatus (JXA-733, JEOL, Tokyo, Japan) operating with an accelerating voltage of $15 \mathrm{kVp}$ and specimen current of $2 \times 10^{-8} \mathrm{~A}$. A line-scan analysis of calcium and fluoride was done on a line running across the cavity wall at the demineralized area in the lesion and the intact area underneath the lesion shown in Fig. 2.

\section{RESULTS}

The shear bond strengths to enamel and dentin surfaces after storage for 24 hours 
are summarized in Table 2. As all specimens of $G$ and $R G$ showed no adhesion to enamel and dentin, those specimens were described as ND (not detected) in Table 2. The bond strength of enamel in the ARG group showed almost the same mean as the ACG group. The bond strength to dentin in the ACG group was significantly lower than that of the ARG group, which showed a mean strength of $6.6 \mathrm{MPa}$ (t-test, $\mathrm{P}<0.05$ ).

Tables 3 and 4 indicate the number of samples classified in the "presence of caries

Table 2 Mean shear bond strengths to enamel and dentin ( $\mathrm{MPa})$

\begin{tabular}{|c|c|c|}
\hline Group & Enamel & Dentin \\
\hline ARG & $11.7(3.3)$ & $6.6(3.1)$ \\
\hline$G$ & ND & ND \\
\hline $\mathrm{RG}$ & ND & ND \\
\hline ACG & $11.1(4.8)$ & $2.4(1.7)$ \\
\hline
\end{tabular}

Mean (SD), $\mathrm{n}=6$

$\mathrm{ND}=$ not detected

*: The means are significantly different between the two groups. $(t-$ test, $\mathrm{p}<0.05)$

ARG: Lining with AB2 \& RES

G: No lining

RG: Lining with RES

ACG: Lining with AB2 \& ABC

Table 3 Number of samples showing caries inhibition zones at enamel walls

\begin{tabular}{lccccccc}
\hline \multirow{2}{*}{ Inhibition zone } & \multicolumn{2}{c}{ Group 1 } & \multicolumn{2}{c}{ Group 2 } & \multicolumn{2}{c}{ Group 3 } \\
& G & ARG & RG & ARG & ACG & ARG \\
\hline Strongly Inhibited & 0 & 4 & 7 & 6 & 1 & 8 \\
Moderately Inhibited & 1 & 2 & 1 & 1 & 2 & 0 \\
Not Inhibited & 7 & 2 & 0 & 1 & 5 & 0 \\
& $\vdots$ & $*$ & & $*$ & & $\vdots$ & $*$ \\
\hline
\end{tabular}

*: Significant difference by Chi-square analysis $(\mathrm{p}<0.05)$

(Number of samples in each group $=8$ )

Table 4 Number of samples showing caries inhibition zones at dentin walls

\begin{tabular}{lccccccc}
\hline \multirow{2}{*}{\multicolumn{1}{c}{ Inhibition zone }} & \multicolumn{2}{c}{ Group 1 } & \multicolumn{2}{c}{ Group 2 } & \multicolumn{2}{c}{ Group 3 } \\
& G & ARG & RG & ARG & ACG & ARG \\
\hline Strongly Inhibited & 1 & 8 & 7 & 8 & 0 & 8 \\
Moderately Inhibited & 5 & 0 & 1 & 0 & 4 & 0 \\
Not Inhibited & 2 & 0 & 0 & 0 & 4 & 0 \\
& $\vdots$ & $*$ & & & $*$ & & $*$ \\
\hline
\end{tabular}

*: Significant difference by Chi-square analysis $(\mathrm{p}<0.05)$

$($ Number of samples in each group $=8$ ) 
Enamel Wall

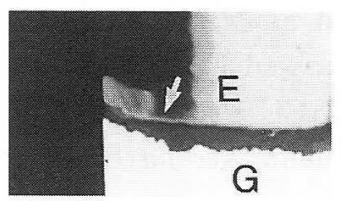

Dentin Wall

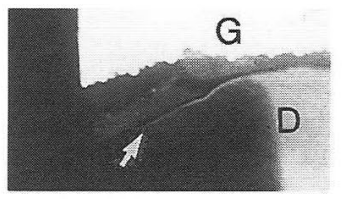

A: Experimental Cavity (ARG)

Enamel Wall

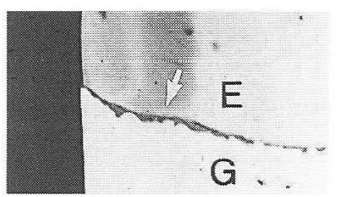

Dentin Wall

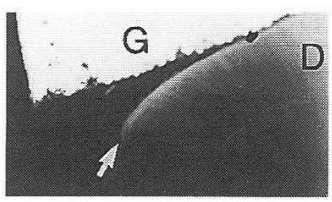

C: Control Cavity (RG)
Enamel Wall

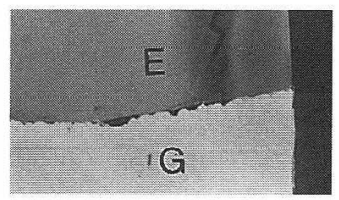

Dentin Wall

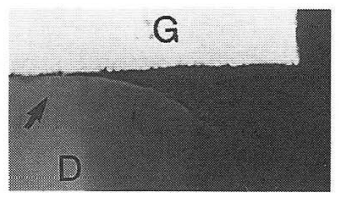

B: Control Cavity (G)

Enamel Wall

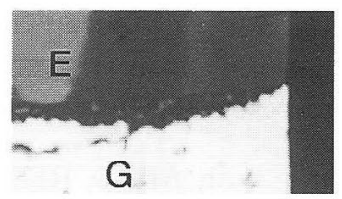

Dentin Wall

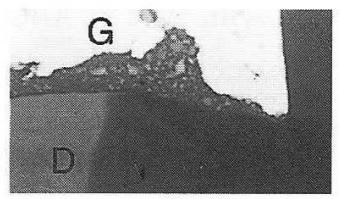

D: Control Cavity (ACG) $\overline{100 \mu \mathrm{m}}$

Fig. 3 Microradiographs of the typical lesions.

E: Enamel D: Dentin G: Gallium alloy restoration

The caries inhibition zones (white arrows) were detected, and were classified as strongly inhibited at the both enamel and dentin lesions adjacent to the RG and ARG cavities.

Although a wall lesion (black arrow) was detected at the dentin lesion adjacent to the G cavity, the caries inhibition zones were detected as slight radiopacities and were classified as moderately inhibited.

Caries inhibition zones were not detected at the enamel lesions adjacent to the $G$ cavities and enamel and dentin lesions adjacent to the ACG cavities were classified as not inhibited.

inhibition' zones at the enamel and dentin walls. At both the enamel walls and dentin walls, comparison of the inhibition zones showed that group $G$ was statistically different from the ARG group, ACG was different from $A R G$, and $R G$ was different from ACG (Chi-square test at $\mathrm{P}<0.05$ ). Caries inhibition zones appeared in all samples at the enamel walls adjacent to RG of group 2 and ARG of group 3, and at the dentin walls next to RG of group 2 and ARG of all groups.

Fig. 3 shows the typical appearance of lesions in each group by microradiography. 
Enamel Wall

Dentin Wall

$\operatorname{CaK} \alpha$ FK $\alpha$
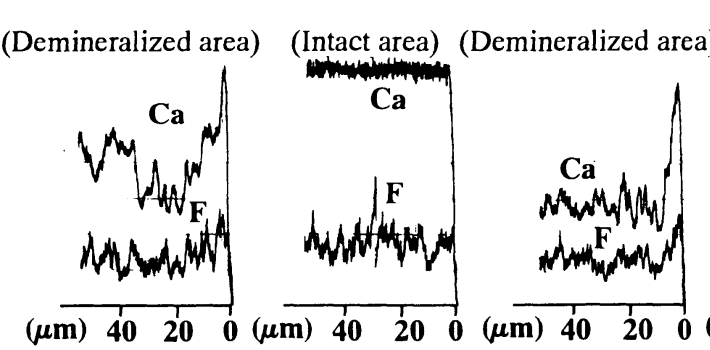

CPS CPS

A:Experimental Cavity (ARG)

Enamel Wall

(Demineralized area)

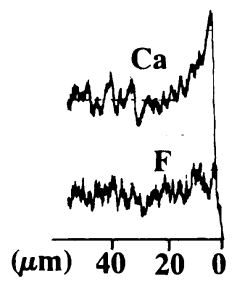

Dentin Wall

(Demineralized area) (Intact area)

(Intact area)
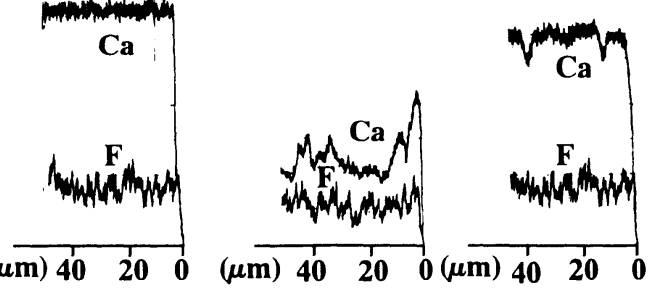

(Intact area)

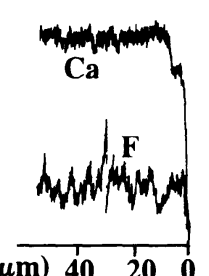

\begin{tabular}{l|l}
$\mathrm{X} 10^{3}$ & $\mathrm{X} 10$
\end{tabular}

B:Control cavity (G)
Bm)

CaK $\alpha$ FK $\alpha$

CPS CPS

$\left|\mathbf{X 1 0}^{3}\right| \mathbf{X 1 0}$

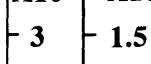

$-2-1$

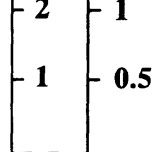

Enamel Wall

Dentin Wall

CaK $\alpha$ FK $\alpha$

(Demineralized area)

(Intact area)

(Demineralized area)

CPS CPS
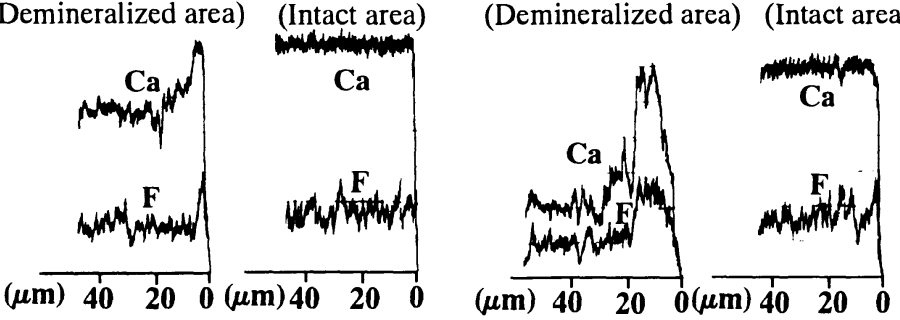

$\times 10^{3} \times 10$

$(\mu \mathrm{m}) 40 \quad 20 \quad 0(\mu \mathrm{m}) 4020 \quad 0$

$(\mu \mathrm{m}) 4020 \quad 0(\mu \mathrm{m}) 40 \quad 20 \quad 0$

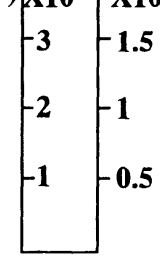

C:Control cavity (RG)

Enamel Wall

(Demineralized area) (Intact area)

Dentin Wall

CaK $\alpha$ FK $\alpha$

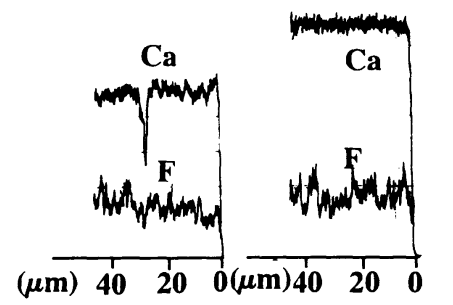

(Demineralized area) (Intact area)

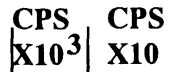

$(\mu \mathrm{m}) \quad 40 \quad 20 \quad 0(\mu \mathrm{m}) 40 \quad 20$

D:Control cavity (ACG)

Fig. 4 Distributions of $\mathrm{Ca}$ and $\mathrm{F}$ counts in the same samples as Fig. 3. 
At the RG control cavity and the ARG experimental cavity, the lesions of the enamel and dentin wall definitely demonstrated caries inhibition zones. In Fig. 3-B, the wall lesion (black arrow) was detected at the dentin lesion adjacent to the cavity restored with $\mathrm{G}$ of group 1. Only the outer lesions could be seen for the ACG control cavity, but caries inhibition zones were not observed (Fig. 3-D).

Fig. 4 shows the line analysis of the above-mentioned sites with the same specimens as Fig. 3. Decreases in calcium content were revealed in the demineralized areas of all enamel and dentin lesions. At the RG control cavity and the ARG experimental cavity, calcium and fluoride counts were high, relating to the caries inhibition zones. Particularly, the calcium peaks in the inhibition zones of demineralized areas were the same high level as those in intact areas at the RG control cavity and the ARG experimental cavity.

\section{DISCUSSION}

If restorations have poor marginal integrity, marginal gaps may extend deeper into the interface and develop secondary caries with not only outer lesions but also wall lesions. The strategy for secondary caries inhibition around amalgam restorations is usually one of the following: 1 . Reinforcement of cavity walls by treatment with fluoride ${ }^{12)} ; 2$. Incorporation of fluoride in amalgam ${ }^{13,14}$; and 3 . Bonding of amalgam to tooth structures treated by an adhesive agent $t^{6)}$. In this study, we studied application of fluoride-releasing liners with bonding of amalgam to tooth structure.

AB2 and RES achieved the highest shear bond strength of amalgam and gallium alloy restoration to dentin among the various bonding systems and liner materials ${ }^{9,15)}$, and produced more effective reduction of microleakage than adhesive resin ${ }^{16)}$. In this study, the bonding strength to dentin using $A B 2$ and RES showed a high mean value of $6.6 \mathrm{MPa}$. Further evidence that AB2 and RES has good adhesion to dentin can be seen from the findings that RES adhered to the dentin wall in the sample from the ARG group (Fig. 3-A). However, the bond strength to enamel and dentin using only RES was not measurable. RES is a Bis-GMA filled resin cement containing sodium-calcium-almino-fluorosilicate glass, and has no chemical bonding reaction to tooth substrates. Therefore, it is important to note that only the combination of RES with $\mathrm{AB} 2$ produced a significant bond of the gallium alloy restoration.

The shear bond strength of $\mathrm{AB} 2$ and $\mathrm{ABC}$ to dentin had a relatively low mean value of $2.4 \mathrm{MPa}$ for gallium alloy restoration in this study. In the examination of failure, most of the debonded enamel and dentin surfaces did not show residues of $\mathrm{ABC}$ and GA. Ružičková et al. ${ }^{4)}$ reported that $\mathrm{AB} 2$ and $\mathrm{ABC}$ achieved high shear and tensile bond strengths to amalgam of more than $8 \mathrm{MPa}$. In the present study, it appears that the failures occurred at the enamel or dentin-ABC interfaces.

Table 3 and 4 showed that the presence of fluoride incorporated in the lining cement protected the marginal tooth structures from acid. The caries inhibition zones in the RG and ARG groups were clearly demonstrated in Fig. 3-A and C. RES contains sodium-calcium-almino-fluorosilicate glass in the base, and gradually releases 
the same amount of fluoride in the long term as several resin-modified glass ionomers for luting ${ }^{17)}$. Nagamine et $a l^{7)}$ reported that high calcium and fluoride counts are related to the thickness of the caries inhibition zones in the demineralized dentin wall adjacent to restorative resin-modified glass ionomers. In Fig. 4-C, high calcium counts were noted at the caries inhibition zones in enamel and dentin, corresponding to a high count of fluoride. The peak levels of calcium in the inhibition zones corresponded with the intact areas. Consequently, fluoride released from RES may be incorporated into the tooth structure surrounding the restoration margins, providing inhibition of calcium dissolution from acid attack.

It was observed that the high calcium counts in the inhibition zone at the $R G$ dentin cavity wall extended deeper than at the ARG dentin cavity wall. 2\% NTGGMA (N-tolyglycine glycidyl methacrylate) and 16\% BPDA (Biphenyl dimethacrylate) included in $\mathrm{ABP}$ may interfere with the migration of fluoride ions. On the other hand, 3 of 8 specimens at the ACG enamel cavity and 4 of 8 specimens at the ACG dentin cavity were classified as having strongly or moderately inhibited caries inhibition zones. The peak calcium count in the inhibition zone of the ACG dentin cavity was lower than that in the intact areas (Fig. 4-D). The resin monomers penetrated into the cavity surface etched by $10 \%$ phosphoric acid gel, and the resin-penetrated layer, the so-called hybrid layer, may inhibit calcium dissolution by demineralization. Thus, these findings suggested that etching and bonding alone were not as effective as fluoride release.

Four of eight ACG dentin cavity specimens were classified as having moderately inhibited zones, while the zones in 6 of $8 \mathrm{G}$ dentin cavity specimens were classified as being strongly or moderately inhibited. The marginal seal at the gallium alloy-tooth interface depends upon good physical properties, and also is correlated with a dimensional change of $0.07 \%{ }^{18)}$. Although some wall lesions were detected at the dentin lesions adjacent to the cavities restored with G (see black arrow in Fig. 3-B), a low peak calcium count in the demineralized dentin area was detected (Fig. 4-B). The good marginal seal resulted in caries inhibition at the gallium alloy-tooth interface. The use of a bonded resin liner reduced microleakage between tooth structure and amalgam restorations significantly more than no varnish and liner ${ }^{19}$. Therefore, it appears that caries inhibitory ability and microleakage are related to the marginal integrity of gallium alloy restorations.

Potential benefits of AB2 and RES would be improvement of marginal seal, and retention and reinforcement of tooth structure. However, the long term clinical effectiveness of the restoration in this system has not been reported and some potential problems would have to be solved: it is difficult to prevent discoloration of the gallium alloy restoration in clinical use and the bonding materials may be incorporated into the gallium alloy, weakening the marginal areas around the restorative materials. Gamou et al. ${ }^{20)}$ reported that the wear resistance of RES was similar to that of conventional glass ionomer luting cement for resin inlay restorations. Clinical observation of marginal areas of gallium alloy restorations with $A B 2$ and RES is needed. 


\section{CONCLUSIONS}

1. AB2 with RES achieved reasonable adhesion of gallium alloy restoration to enamel and dentin, but RES alone had no measurable bonding to tooth substrates.

2 . The caries inhibition zones of enamel and dentin walls adjacent to gallium alloy restorations were clearly visible when RES was used as a cavity liner with or without the AB2 bonding system.

3 . The counts of calcium in the inhibition zones were comparable to the counts in intact areas.

4. The results indicated that the fluoride releasing resin-ionomer cement provided good adhesion with etching and bonding and caries inhibition with or without etching and bonding, and that etching and bonding alone were not as effective as fluoride release.

\section{ACKNOWLEDGMENTS}

The authors wish to thank Mr. Naruse of Tokuriki Honten Co., Ltd. for supplying the test materials used in this study, and acknowledge the technical assistance of Associate Professor Chiyoko Henmi of the Department of Earth Sciences, Faculty of Science, Okayama University, for the electron probe microanalysis.

\section{REFERENCES}

1) Hals, E. and Simonsen, T. L.: Histopathology of experimental in vivo caries around silver amalgam fillings, Caries Res $6: 16-33,1972$.

2) Goldberg, J., Amara, J., Tanzer, J., Thal, F., Munster, E. and Birkhed, D.: Crosssectional clinical evaluation of recurrent enamel caries, restoration of marginal integrity, and oral hygiene status, JADA $102: 635-641,1981$.

3) Kidd, E. A. M. and O'hara, J. W.: The caries status of occlusal amalgam restorations with marginal defects, $J$ Dent Res $69:$ 1275-1277, 1990.

4) Ružičková, T., Staninec, M., Marshall, G. W. and Hutton, J. E.: Bond strengths of the adhesives resin-amalgam interface, Am J Dent 10(4) : 192-194, 1997.

5) Berry, F. A., Parker, S. D., Rice, D. and Muñoz, C. A.: Microleakage of amalgam restorations using dentin bonding system primers, Am J Dent 9 : 174-178, 1996.

6) Torii, Y., Staninec, M., Kawakami, M., Imazato, S., Torii, M. and Tsutitani, Y.: Inhibition in vitro of caries around amalgam restorations by bonding amalgam to tooth structure, Oper Dent $14: 142-148,1989$.

7) Nagamine, M., Itota, T., Torii, Y., Irie, M., Staninec, M. and Inoue, K.: Effect of resinmodified glass ionomer cements on secondary caries, Am J Dent $10: 173-178,1997$.

8) Al-Moayad, M., Aboush, Y.E. Y. and Elderton, R. J.: Bonded amalgam restorations: a comparative study of glass-ionomer and resin adhesives, Brit Dent J $175: 363-367,1993$.

9) Ng, B. P., Purton, D. G. and Hood, J. A. A.: Effects of lining materials on shear bond strength of amalgam and gallium alloy restorations, Oper Dent 23(3): 113-120, 1998.

10) Mackert, J.R., Jr. and Berglund, A.: Mercury exposure from dental amalgam fillings: absorbed dose and the potential for adverse health effects, Crit Rev Oral Biol Med 8(4): 410-436, 1997.

11) Horibe, T., Okamoto, Y. and Naruse, S.: Gallium alloys for dental restorations Part1. Physical properties of gallium alloys, J Fukuoka Dent Coll 12(4):198-204, 1986. (in 
Japanese)

12) Inoue, K., Tanizaki, K., Teraoka, K. and Kawagoe, M.: In vitro studies of recurrent caries VIII. Effect of stannous fluoride on the reduction of recurrent caries around the amalgam restoration, Japan J Conserv Dent 19(2):303-309, 1976. (in Japanese)

13) Skartveit, L., Wefel, J.S. and Ekstrand, J.: Effect of fluoride amalgams on artificial recurrent enamel and root caries, Scand $J$ Dent Res 99(4) : 287-294, 1991.

14) Valenzuela, V.S., Abarca, A. M., Silva, N. D. C., Franco, M. E. and Huerta, J. M.: In vitro inhibition of marginal caries-like lesions with fluoride-containing amalgam, Oper Dent 19(3): 91-96, 1994.

15) Kline, J. and Boyer, D.: Bond strength of amalgam to dentin with filled adhesives, $J$ Dent Res 75 (Special issue) : 175, 1996.

16) Tangsgoolwatana, J., Cochran, M. A., Moore, B. K. and Li, Y.: Microleakage evaluation of bonded amalgam restorations: Confocal Microscopy versus radioisotope, J Dent Res 75 (Special Issue) : 172, 1996.

17) Burgess, J. O.: Dental materials for the restoration of root surface caries, Am $J$ Dent $8: 342-351,1995$.

18) Okamoto, Y., Naruse, S. and Yamamoto, H.: Influence of $\mathrm{Pd}$ addition on the physical properties of gallium filling materials, J Fukuoka Dent Coll 19(4):347-352, 1992. (in Japanese)

19) Staninec, M. and Holt, M.: Bonding of amalgam to tooth structure: Tensile adhesion and microleakage tests, $J$ Prosthet Dent 59(4):397-402, 1988.

20) Gamou, K., Hama, K., Nagamine, M., Staninec, M., Torii, Y., Irie, M. and Inoue, K.: Wear resistance of resin-modified glass ionomer cements, $J$ Dent Res 77 (Special issue) : 688, 1998. 